\title{
SOLUTION OF THE EQUATION OF SECULAR VARIATION BY A METHOD DUE TO HERMITE
}

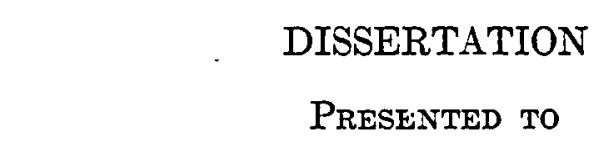

The Faculty of the University of Virginta

BY

EDWARD STAPLES SMITH

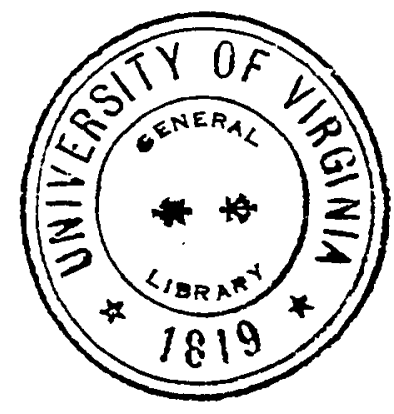

PRESS OF
THE NEW ERAPRINTING COMPANY

1916 


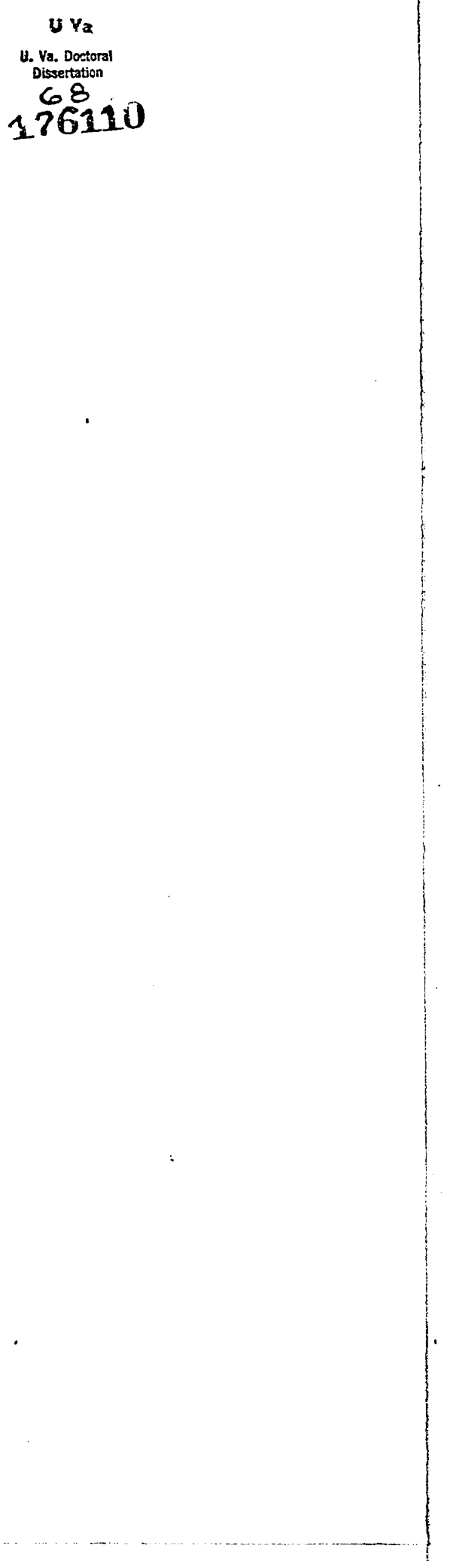




\section{PREFACE}

The "Equation of Secular Variations" plays such an important rôle in many analytical investigations, particularly in the determination of the secular variations of the planets, and in fixing the principal axes of surfaces of the second degree, that Dr. Hancock suggested I undertake its solution by "Hermite's Method."

This is done in the present paper, which has been developed in three distinct parts, as follows:

I. A History of the Secular Variations of the Planets, Introducing the Secular Equation.

II. The Development of "Hermite's Method."

III. The Solution of the Equation of Secular Variations and Applications.

The history has been obtained largely from Die Mathematischen Theorien der Planeten-Bewegungen, von Dr. Otto Dziobek, from Laplace's Collected Works, Vol. XI, page 49, and from Moulton's Celestial Mechanics. No special reference is given in the history to the one of the three authors from whom the material was obtained, as they were all used throughout. The history necessarily deals-with the other perturbations as well as the secular terms, but I have attempted to lay special stress on the history of the secular, rather than the periodic, terms.

In the development of the form of Sturm's Theorem known as "Hermite's Method of Solving Sturm's Theorem"Weber's Algebra has been closely followed. In order to have the method before me in concise form for ready reference, and to develop the method in logical order, I have tabulated in full the material needed to derive the Method.

In the third chapter, application is made of the method set forth in Chapter II, and the equation is solved independently of any previous solution. The solution is then followed by a few important applications.

Besides the general credit given above for the source of my material, an attempt has been made to give due credit in footnotes.

I wish to acknowledge here my indebtedness to Professor W. H. Echols, and Dr. J. M. Page, who labored with me as a student at the University of Virginia to make it possible for me to pursue further investigations, and also to thank Dr. Harris Hancock for suggesting the problem.

University of Cincinvats, March 15, 1916. 


\section{CHAPTER I}

\section{A Short History of the Perturbations of the Planets, with Particular Attention to the Secular Terus}

The Equation of Secular Variations, the solution of which is the object of this treatise, is so named because of its part in establishing the secular terms in the perturbations of three or more planets.

According to Laplace,* the planetary perturbations divide themselves into two classes, the periodic and the secular.

The periodic are, for the most part, very small, depend on the positions of the planets in their orbits, and usually run their course in a few revolutions of the planet in question.

In the earlier investigations the secular inequalities were not considered, as their influence is negligible unless exerted over a very great period, and they were not considered until it was demonstrated that over cycles of centuries their influence is enormous. These inequalities are not dependent on the position of the planets in their orbits, but on the relative positions of the orbits themselves.

The history of the disturbance theories begins with Newton, who explained the principal irregularities in the movement of the moon in the third book of his Principia. He developed the theory of perturbations as applied to the Lunar Theory by the methods of geometry.

Clairaut and D'Alembert made further progress in their memoirs of 1747 , presented to the French Academy of Sciences on the same day, in which they solved the problem of three bodies when the central force outweighs the remaining in importance. Their solution depended on the integration of the differential equation of motion in series. Clairaut soon made a forceful application of his brilliant theory, by studying the perturbations of Halley's Comet. This comet had been observed in 1531, 1607 and 1682 and its period fixed as about seventy-five years. Its return was therefore expected at the latest toward the end of the year 1758. Clairaut calculated the influence which the then known planets would exercise on it, and reported to the Academy of Sciences that the disturbance caused by them would delay its return until the middle of April, 1759. He later added this might vary about a month due to small influences he had neglected. While the comet reappeared within the limit given by him, being first seen on March 12, 1759, still there was a greater

* See Laplace's Collected Works. 
difference between his calculation and the actual occurrence than there would have been had the mass of Saturn been more correctly known.

The methods of geometry were succeeded by the more powerful methods of analysis, and were developed largely by Euler, Clairaut, D'Alembert, Lagrange and Laplace.

Euler (1707-1783) was the pioneer in the applications of analysis to the perturbations, having to develop the methods which he used in attacking the problem. He presented three memoirs on the perturbations of the planets, treating primarily the mutual perturbations of Jupiter and Saturn, which gained for him the prizes of the Paris Academy of Sciences in the years 1748, 1752 and 1756 . In these memoirs he gave the first analytical development of the method of the variations of parameters. His formulas were not entirely general because he did not vary many of the constants simultaneously. Still, in the case of two planets he arrived at the proof of the existence of the secular variations of the eccentricity, inclination, perihelion, and node. Unfortunately many mistakes appeared in the numerical calculations, but the results were later correctly obtained by Lagrange.

Lagrange (1736-1813) pursued the ideas of Euler, and developed still further the method of the variation of parameters. His contributions to celestial mechanics were exceptionally valuable, and should insure him a permanent monument in all works on astronomy. His first work appeared in the Mélanges de la Société de Turin, Tome III, 1766. In this he applied the methods of the variations of parameters to the perturbations of Jupiter and Saturn, but his procedure was not precise because he regarded the major axes and the epochs of the perihelion passages as constants. However, limiting himself to two planets, he obtained the right final equation for the consideration of the secular periods. This had been given incorrectly by Euler. For the inclinations and nodes he obtained correct formulas, so that his numerical results, by the use of the theory of the greatest planets-Jupiter and Saturnare passably correct.

In the year 1773 Laplace (1749-1827) presented to the Academy of Sciences his first work on the theory of the planetary system, which appeared in the year 1776 in the Mémoires des Savants étrangers. He fixed with full accuracy the formula for the differential quotient of the elements, though this did not at first possess its later elegant form. In this memoir he proved his celebrated theorem that, up to the second powers of the.eccentricities and the inclinations, the major axes have no secular terms.

The theory of the secular variations of the elements arose because in the customary procedure of the integration through series, terms were produced which were proportional to the time. Euler, Lagrange and Laplace now concentrated their reflections on the effort to remove these terms. Soon the 
self-evident fact that the theory of absolute disturbances of the planetary system was not the most suitable, caused the theory of the variations of the constants to be introduced, not at first fully clear, but mixed with the former theory.

It was evident that the secular terms must endure, but instead of determining it directly it was grasped in a roundabout way. This was clearly seen in Laplace's Mécanique Cêleste where, in the expression for the absolute disturbance of the coördinates, he ingeniously withdrew the terms which were proportional to the time, and then after the introduction of the full theory of the variation of the constants to the integration he found the secular terms of the disturbance theorem retained.

Poisson proved in 1809 , in the treatise "Sur les inégalités séculaires des moyens movements des planètes" (Journal de l'école polytechnique), that the major axes have no purely secular terms in the perturbations of the second order with respect to the masses. He arrived at his results by a peculiar application of the theorem of active forces. Laplace, in his Mécanique Céleste, livre VI, gave another proof, and Lagrange had sought to furnish this proof but Serret has shown that his procedure suffered a slight mistake in sign.

It was sought to extend this result to the third power of the disturbing masses. Thus Mathiew, in his "Memoire sur les inégalités séculaires des grands axes des orbits des planètes" (Journal von Crelle, 1875), began investigations from which he concluded that the secular terms did not appear even in the consideration of the third powers. But finally, Harétu proved, in his Dissertation at Sorbonne in 1878 , that there are secular variations in the expressions for the major axes in the terms of the third order with respect to the masses.

The analytical and numerical development of the disturbance functions, when considering higher powers of the eccentricity and inclination, led to such interesting and important computations that the majority of the important mathematicians have taken pains with it and advanced it. Among these we find Cauchy, Bessel, Lubback, Hansen, Glyden, Newcomb and others. Gauss, Airy, Adams, Leverrier and many others have made important contributions to the planetary theory in some of its many aspects. Adams and Leverrier obtained particular prominence by demonstrating the existence of Neptune and calculating its apparent position from the unexplained irregularities in the motion of Uranus.

The secular value of the elements had been developed by Laplace and Lagrange, limiting their discussion to the second power of the eccentricity and inclination. According to Dziobek it was Leverrier who first attempted to consider the influence of the neglected terms. Later his investigations were carried further by the astronomer Lehmann, who, however, died before com- 
pleting his numerical investigation. It was the opinion of Leverrier and Lehmann that the influence of these terms is greater than was earlier supposed, and would possibly bring great improvement on the secular periods, as they would be fixed through the roots of the secular equation.

\section{The Sectlar Equation}

The way the secular equation enters is shown by the following treatment taken from Dziobek.*

The secular part of the disturbance functions depends on the quantity

$$
W=\Sigma \frac{m_{\lambda} m_{\mu}}{r_{\lambda \mu}}
$$

When limiting the secular part, $W$, of the disturbance function to the terms of second degree of the eccentricity and inclination, $W$ reduces to

$$
\begin{aligned}
\dagger W=\Sigma m_{\lambda} m_{\mu}\left[\frac{1}{2} I_{0}+\frac{1}{8} I I I_{1}\left\{h_{\lambda}^{2}+h_{\mu}^{2}\right.\right. & +l_{\lambda}^{2}+l_{\mu}^{2}-\left(p_{\lambda}-p_{\mu}\right)^{2} \\
& \left.\left.-\left(q_{\lambda}-q_{\mu}\right)^{2}\right\}-\frac{1}{4} I I I_{2}\left(h_{\lambda} h_{\mu}+l_{\lambda} l_{\mu}\right)\right] .
\end{aligned}
$$

The differential equations of motion then become .

$$
\begin{aligned}
& m_{\lambda} \frac{d h_{\lambda}}{d t}=\frac{1}{\sqrt{\mu_{\lambda} a_{\lambda}}} \cdot \frac{\partial W}{\partial l_{\lambda}}, \\
& m_{\lambda} \frac{d l_{\lambda}}{d t}=-\frac{1}{\sqrt{\mu_{\lambda} a_{\lambda}}} \cdot \frac{\partial W}{\partial h_{\lambda}} . \\
& m_{\lambda} \frac{d p_{\lambda}}{d t}=\frac{1}{\sqrt{\mu_{\lambda} a_{\lambda}}} \cdot \frac{\partial W}{\partial q_{\lambda}}, \quad(\lambda=1,2, \cdots, n) . \\
& m_{\lambda} \frac{d q_{\lambda}}{d t}=-\frac{1}{\sqrt{\mu_{\lambda} a_{\lambda}}} \cdot \frac{\partial W}{\partial p_{\lambda}} .
\end{aligned}
$$

Substitute the value of $W$ in (2) in these differential equations, limiting to terms in $h$ and $l$, and the result can be expressed in two groups, those containing only $h$, and those containing only $l$. The two groups are brought down in final form to a quadratic form in $H_{\lambda}$ and $L_{\lambda}$ (defined in (8), page 225). These quadratic forms when transformed by linear substitutions into a sum of squares, finally reduce to the set of equations, +

* Driobek, Planelen-Bercegungen, pages 221 to 227.

$\dagger(2), \S 31$, page 224.

$\ddagger(20)$, page 227 . 


$$
\begin{aligned}
& 0=\alpha_{1,1}\left([1,1]-g_{1}\right)+\alpha_{2,1}[2,1]+\alpha_{3,1}[3,1]+\cdots+\alpha_{n, 1}[n, 1] \\
& 0=\alpha_{1,1}[1,2]+\alpha_{2,1}\left([2,2]-g_{1}\right)+\alpha_{3,1}[3,2]+\cdots+\alpha_{n, 1}[n, 2] \\
& 0=\alpha_{1,1}[1,3]+\alpha_{2,1}[2,3]+\alpha_{3,1}\left([3,3]-g_{1}\right)+\cdots+\alpha_{n, 1}[n, 3] \\
& 0=\alpha_{1,1}[1, n]+\alpha_{2,1}[2, n]+\alpha_{3,1}[3, n]+\cdots+\alpha_{n, 1}\left([n, n]-g_{1}\right) .
\end{aligned}
$$

The $g_{\lambda}$ in these equations give the coefficients of the square terms when expressed as sums of squares. The $n g$ 's are obtained by eliminating from the equations (20) the unknown

when there results

$$
\alpha_{1,1}, \alpha_{2,1}, \alpha_{3,1}, \cdots, \alpha_{n, 1},
$$

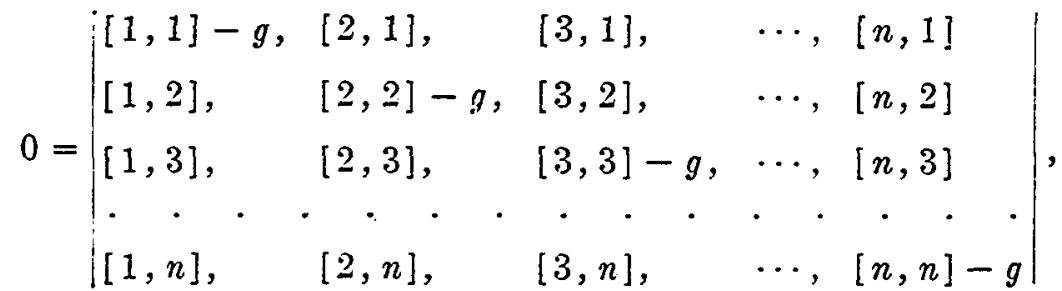

the roots of which give us the $g$ 's. 


\section{CHAPTER II}

\section{Hermite's Method of Solving SturM's Theorem}

$\S 1$. Before passing to the solution of our particular problem we shall develop or tabulate, for convenience of reference, the method known as "Hermite's Solution of Sturm's Theorem."* This method leads frequently to a simpler result than is obtained by direct application of Sturm's Theorem, and is closely related to Hermite's work with the Tschirnhausen's Transformations. It also depends or the theorem in quadratic forms called by Sylvester $\dagger$ "The Law of Inertia of Quadratic Forms" which states:

"If we express a real quadratic form $\varphi(x)$ as a sum of positive and negative squares of linear functions (which may be done in an infinite number of ways) then the number of positive and of negative squares, as also of their sum, will always be the same, provided there is no linear relation between the functions."

\section{§2. The Quadratic Form $H_{a}$. Consider the equation}

$$
f(x)=a_{0} x^{n}+a_{1} x^{n-1}+a_{2} x^{n-2}+\cdots+a_{n-1} x+a_{n}=0,
$$

whose roots,

$$
x_{1}, x_{2}, \cdots, x_{n},
$$

we shall assume are all unequal.

If $x$ is a root of (1) we can build the expression

$$
\frac{f(t)}{t-x}=t^{n-1} f_{0}(x)+t^{n-2} f_{1}(x)+\cdots+t f_{n-2}(x)+f_{n-1}(x),
$$

in which $t$ is an undetermined quantity.

The coefficients of the powers of $t$ are here

$$
\begin{aligned}
f_{0}(x) & =a_{0}, \\
f_{1}(x) & =a_{0} x+a_{1}, \\
f_{2}(x) & =a_{0} x^{2}+a_{1} x+a_{2}, \\
\cdot & \cdot \cdot \cdot \cdot \cdot \cdot \cdot \cdot \cdot \cdot \cdot \\
f_{n-1}(x) & =a_{0} x^{n-1}+a_{1} x^{n-2}+a_{2} x^{n-3}+\cdots+a_{n-2} x+a_{n-1} .
\end{aligned}
$$

* Hermite, " Remarques sur le théorème de M. Sturm," Comptes rendus de Paris Akademie, T. 30 (1853).

† See Philosophical Magazine, 1852. 
Now construct the function

$$
y=t_{n-1} f_{0}(x)+t_{n-2} f_{1}(x)+\cdots+t_{1} f_{n-2}(x)+t_{0} f_{n-1}(x),
$$

in which $t_{0}, t_{1}, \cdots, t_{n-1}$ are undetermined constants, and are not to be confused with powers of $t$. However (4) goes into (2) if $t_{k}$ is changed to $t^{h}$.

Denoting by

$$
y_{1}, y_{2}, y_{3}, \cdots, y_{n}
$$

the values which $y$ takes for

$$
x=x_{1}, x_{2}, x_{3}, \cdots, x_{n}
$$

respectively and taking $\alpha$, any fixed real quantity, we next build the expression

$$
H_{\alpha}=\left(x_{1}-\alpha\right) y_{1}^{2}+\left(x_{2}-\alpha\right) y_{2}^{2}+\cdots+\left(x_{n}-\alpha\right) y_{n}^{2} \text {. }
$$

This is a quadratic form in the $n$ variables $t_{i}$, and below it will be shown that the number of its negative terms, for a constant $\alpha$, gives the number of real roots of $f(x)=0$ which are less than $\alpha$, plus the number of pairs of imaginary roots of $f(x)=0$.

If $x_{1}$ is a real root, then $y_{1}$ is real, and the term

$$
\left(x_{1}-\alpha\right) y_{1}^{2}
$$

is positive or negative according as $x_{1}$ is greater or less than $\alpha$. When $x_{1}$ and $x_{2}$ form a pair of conjugate imaginaries, then $y_{1}$ and $y_{2}$ are conjugate imaginaries and

$$
\left(x_{1}-\alpha\right) y_{1}^{2}+\left(x_{2}-\alpha\right) y_{2}^{2}
$$

is composed of a positive and a negative square. This is easily seen if we take

whence

$$
y_{1} \sqrt{x_{1}-\alpha}=u+i v, \quad y_{2} \sqrt{x_{2}-\alpha}=u-i v,
$$

$$
\left(x_{1}-\alpha\right) y_{1}^{2}+\left(x_{2}-\alpha\right) y_{2}^{2}=2 u^{2}-2 v^{2} \text {. }
$$

From the foregoing it follows that the number $N_{a}$ of the negative squares in $H_{a}$ is equal to the total number of pairs of imaginary roots of $f(x)=0$ plus the number of its real roots which are less than $\alpha$.

Take now a second real number $\beta>\alpha$, and form the function $H_{\beta}$, representing by $N_{\beta}$ the number of negative squares of $H_{\beta}$. Then the difference

$$
N_{\beta}-N_{\alpha}
$$

is equal to the number of real roots of $f(x)=0$ lying between $\alpha$ and $\beta$.

§3. Some Relations between the Coefficients. We have thus a means of determining the real roots of an equation, if we can represent the coefficients of the $t_{i} t_{j}$ in the function $H_{\alpha}$ as functions of $\alpha$ and of the coefficients of the 
powers of $x$ in $f(x)$. After a few expressions, which are used in developing the process or in its application, are derived, then we shall proceed to determine these coefficients. The symbol $S$ in front of an expression is used here to indicate the expression is to be summed over all the roots of $f(x)=0$. Thus

$$
\begin{aligned}
& S(x)=x_{1}+x_{2}+\cdots+x_{n}, \\
& S(y)=y_{1}+y_{2}+\cdots+y_{n} .
\end{aligned}
$$

Summing both sides of (2) over the roots of $f(x)=0$ there results the expressions,

$$
\begin{aligned}
& S\left[f_{0}(x)\right]=n a_{0}, \\
& S\left[f_{1}(x)\right]=(n-1) a_{1}, \\
& S\left[f_{2}(x)\right]=(n-2) a_{2}, \\
& \cdot \cdot \cdot \cdot \cdot \cdot \cdot \\
& S\left[f_{n-1}(x)\right]=a_{n-1} .
\end{aligned}
$$

The left side of (2) gives

$$
\begin{aligned}
S\left(\frac{f(t)}{t-x}\right)=\frac{f(t)}{t-x_{1}} & +\frac{f(t)}{t-x_{2}}+\cdots+\frac{f(t)}{t-x_{n}} \\
& =\frac{d}{d t} f(t)=n a_{0} t^{n-1}+(n-1) a_{1} t^{n-2}+\cdots+a_{n-1}
\end{aligned}
$$

and the right side of (2) summed gives

(8) $S\left(\frac{f(t)}{t-x}\right)=t^{n-1} S\left[f_{0}(x)\right]+t^{n-2} S\left[f_{1}(x)\right]+\cdots+S\left[f_{n-1}(x)\right]$.

Comparing coefficients in ( 7$)$ and (8) the expressions in (6) are found.

§4. Determination of Hermite's Form H. Represent the product of $y f_{s}$ by

$$
\begin{aligned}
y f_{s}=E_{0, s} f_{0}+E_{1,8} f_{1}+E_{2, s} f_{2}+\cdots+E_{n-1, s} f_{n-1} \\
s=0,1,2, \cdots, n-1 .
\end{aligned}
$$

When $s=0$, comparing coefficients in (9) and $y f_{0}$ obtained from (4), the set of $E_{i, s}$ for $s=0$ are found to be, since $f_{0}(x)=a_{0}$,

$$
E_{0,0}=a_{0} l_{n-1}, \quad E_{1,0}=a_{0} t_{n-2}, \quad \cdots, \quad E_{n-1,0}=a_{0} t_{0} .
$$

The remaining $E_{i, j}$ functions are determined from the following relations, which are true in virtue of (3). 
(11)

$$
\begin{aligned}
& x f_{0}=a_{6} x=f_{1}-a_{1}, \\
& x f_{1}=f_{2}-a_{2}, \\
& x f_{2}=f_{3}-a_{3},
\end{aligned}
$$

$$
x f_{n-2}=f_{n-1}-a_{n-1},
$$$$
x f_{n-1}=f-a_{n},
$$

since $f(x)=0$.

$$
=-a_{n},
$$

From (9) and (11) we have,

$$
\begin{aligned}
x y f_{s}=E_{0, s} f_{1} & +E_{1, s} f_{2}+\cdots+E_{n-2, s} f_{n-1} \\
& -a_{1} E_{0, s}-a_{2} E_{1, s}-\cdots-a_{n-1} E_{n-2, s}-a_{n} E_{n-1, s} .
\end{aligned}
$$

Now define $E_{-1, \text { s }}$ by

$$
a_{0} E_{-1, s}+a_{1} E_{0, s}+a_{2} E_{1, s}+\cdots+a_{n} E_{n-1, s}=0 .
$$

Multiply (9) by $\alpha$ and subtract from (12) using (13) and we get

$$
\begin{aligned}
(x-\alpha) y f_{s}=\left(E_{-1, s}-\alpha E_{0, s}\right) f_{0}+\left(E_{0, s}-\alpha E_{1, s}\right) f_{1} & +\cdots \\
& +\left(E_{n-2, s}-\alpha E_{n-1, s}\right) f_{n-1} .
\end{aligned}
$$

$E_{-1,8}$ may be expressed from the equation

$$
a_{0} t_{n+s}+a_{1} t_{n+8-1}+\cdots+a_{n} t_{s}=0,
$$

which is obtained by introducing

$$
t_{n}, t_{n+1}, t_{n+2}, \cdots
$$

through the following equations

$$
\begin{aligned}
& a_{0} t_{n}+a_{1} t_{n-1}+a_{2} t_{n-2}+\cdots+a_{n} t_{0}=0, \\
& a_{0} t_{n+1}+a_{1} t_{n}+a_{2} t_{n-1}+\cdots+a_{n} t_{1}=0, \\
& a_{0} t_{n+2}+a_{1} t_{n+1}+a_{2} t_{n}+\cdots+a_{n} t_{2}=0,
\end{aligned}
$$

Multiply equations (11) through, in order, by

and add, whence

$$
t_{n-1}, t_{n-2}, \cdots, t_{0}
$$

$$
x y=t_{n} f_{0}+t_{n-1} f_{1}+\cdots+t_{1} f_{n-1} .
$$

Similarly multiply equations (11) through, in order, by

$$
t_{n}, t_{n-1}, \cdots, t_{1}
$$

add and use (16) and (17), and we have, 


$$
x^{2} y=t_{n+1} f_{0}+t_{n} f_{1}+\cdots+t_{2} f_{n-1} .
$$

Continuing this process the following system is obtained:

$$
\begin{aligned}
y & =t_{n-1} f_{0}+t_{n-2} f_{1}+t_{n-3} f_{2}+\cdots+t_{0} f_{n-1}, \\
x y & =t_{n} f_{0}+t_{n-1} f_{1}+t_{n-2} f_{2}+\cdots+t_{1} f_{n-1}, \\
x^{2} y & =t_{n+1} f_{0}+t_{n} f_{1}+t_{n-1} f_{2}+\cdots+t_{2} f_{n-1}, \\
x^{3} y & =t_{n+2} f_{0}+t_{n+1} f_{1}+t_{n} f_{2}+\cdots+t_{3} f_{n-1}, \\
\cdot & \cdot \cdot \cdot \cdot \cdot \cdot \cdot \cdot \cdot \cdot \cdot \cdot \cdot \cdot \cdot \cdot \cdot \\
x^{s} y & =t_{n+s-1} f_{0}+t_{n+s-2} f_{1}+t_{n+s-3} f_{2}+\cdots+t_{s} f_{n-1} .
\end{aligned}
$$

These relations multiplied through, in order, by

$$
a_{s}, a_{s-1}, a_{s-2}, \cdots, a_{0}
$$

and added give the equation

$$
y f_{s}=E_{0, s} f_{0}+E_{1, s} f_{1}+\cdots+E_{n-1, s} f_{n-1},
$$

in which the $E_{i, s}$ are defined by

$$
\begin{aligned}
& E_{-1, s}=a_{0} t_{n+s}+a_{1} t_{n+8-1}+\cdots+a_{s} t_{n} \\
& =-a_{s+1} t_{n-1}-\cdots-a_{n} t_{s}, \\
& E_{0, s}=a_{0} t_{n+8-1}+a_{1} t_{n+s-2}+\cdots+a_{s} t_{n-1} \\
& =-a_{s+1} t_{n-2}-\cdots-a_{n} t_{s-1}, \\
& E_{1,8}=a_{0} t_{n+s-2}+a_{1} t_{n+s-3}+\cdots+a_{s} t_{n-2} \\
& =-a_{s+1} t_{n-3}-\cdots-a_{n} t_{s-2}, \\
& E_{2, s}=a_{0} t_{n+s-3}+a_{1} t_{n+s-4}+\cdots+a_{s} t_{n-3} \\
& =-a_{s+1} t_{n-4}-\cdots-a_{n} t_{s-3}, \\
& E_{s-1, s}=a_{0} t_{n}+a_{1} t_{n-1}+\cdots+a_{s} t_{n-s} \\
& =-a_{s+1} t_{n-s-1}-\cdots-a_{n} t_{0}, \\
& E_{s, s}=a_{0} t_{n-1}+a_{1} t_{n-2}+\cdots+a_{s} t_{n-s-1}, \\
& E_{s+1, s}=a_{\mathrm{c}} t_{n-2}+a_{1} t_{n-3}+\cdots+a_{s} t_{n-s-2}, \\
& E_{n-1, s}=a_{0} t_{s}+a_{1} t_{s-1}+\cdots+a_{s} t_{0} .
\end{aligned}
$$

Next introduce a second system of variables $\tau$ through

$$
z=\tau_{n-1} f_{0}+\tau_{n-2} f_{1}+\cdots+\tau_{0} f_{n-1},
$$


from which we have, since by (6) $S\left(f_{s}\right)=(n-s) a_{s}$,

$$
\begin{aligned}
& S[(x-\alpha) y z]=S\left[(x-\alpha) y\left\{\tau_{n-1} f_{0}+\tau_{n-2} f_{1}+\cdots+\tau_{0} f_{n-1}\right\}\right] \\
& =S\left[\tau_{n-1}(x-\alpha) y f_{0}+\tau_{n-2}(x-\alpha) y f_{1}+\cdots\right. \\
& \left.\left.+\tau_{0}(x-\alpha) y f_{n-1}\right\}\right] \\
& =n a_{0} \sum_{0, n-1}^{s}\left(E_{-1, s}-\alpha E_{0, s}\right) \tau_{n-s-1} \\
& +(n-1) a_{1} \sum_{0, n-1}^{s}\left(E_{0, s}-\alpha E_{1, s}\right) \tau_{n-s-1} \\
& +a_{n-1} \sum_{0, n-1}^{s}\left(E_{n-2, s}-\alpha E_{n-1, s}\right) \tau_{n-s-1} \text {. }
\end{aligned}
$$

On making $\tau=t(22)$ will take the value of $H_{a}$ in (5) being expressed in terms of the coefficients of $f(x)$ and of $\alpha$.

$\S$ 5. Illustrative Example. For an example take the case $n=4$, for which we find,

$$
\begin{aligned}
& E_{-1,0}=-a_{1} t_{3}-a_{2} t_{2}-a_{3} t_{1}-a_{4} t_{0}, \\
& E_{-1,1}=-a_{2} t_{3}-a_{3} t_{2}-a_{4} t_{1}, \\
& E_{-1,2}=-a_{3} t_{3}-a_{4} t_{2}, \\
& E_{-1,3}=-a_{4} t_{3}, \\
& E_{0,0}=a_{0} t_{3} \\
& E_{0,1}=-a_{2} t_{2}-a_{3} t_{1}-a_{4} t_{0}, \\
& E_{0,2}=-a_{3} t_{2}-a_{4} t_{1}, \\
& E_{0,3}=-a_{4} t_{2}, \\
& E_{1,0}=a_{0} t_{2}, \\
& E_{1,1}=a_{0} t_{3}+a_{1} t_{2} \\
& E_{1,2}=-a_{3} t_{1}-a_{4} t_{0}, \\
& E_{1,3}=-a_{4} t_{1}, \\
& E_{2,0}=a_{0} t_{1}, \\
& E_{2,1}=a_{0} t_{2}+a_{1} t_{1}, \\
& E_{2,2}=a_{0} t_{3}+a_{1} t_{2}+a_{2} t_{1}, \\
& E_{2,3}=-a_{4} t_{0}, \\
& E_{3,0}=a_{0} t_{0} \\
& E_{3,1}=a_{0} t_{1}+a_{1} t_{0}, \\
& E_{3,2}=a_{0} t_{2}+a_{1} t_{1}+a_{2} t_{0}, \\
& E_{3,3}=a_{0} t_{3}+a_{1} t_{2}+a_{2} t_{1}+a_{2} t_{0} .
\end{aligned}
$$


These values of $E_{i, j}$ substituted in (22) give

$$
\begin{gathered}
\begin{aligned}
S[(x-\alpha) y z]= & t_{3} \tau_{3} H_{3,3}+t_{3} \tau_{2} H_{3,2}+t_{3} \tau_{1} H_{3,1}+t_{3} \tau_{0} H_{3,0} \\
& +t_{2} \tau_{3} H_{2,3}+t_{2} \tau_{2} H_{2,2}+t_{2} \tau_{1} H_{2,1}+t_{2} \tau_{0} H_{2,0} \\
& +t_{1} \tau_{3} H_{1,3}+t_{1} \tau_{2} H_{1,2}+t_{1} \tau_{1} H_{1,1}+t_{1} \tau_{0} H_{1,0} \\
& +t_{0} \tau_{3} H_{0,3}+t_{0} \tau_{2} H_{0,2}+t_{0} \tau_{1} H_{0,1}+t_{0} \tau_{0} H_{0,0} .
\end{aligned} \\
\text { If } \tau=t \text { this goes into }
\end{gathered}
$$

$$
\begin{aligned}
H_{a}= & t_{3}^{2} H_{3,3}+t_{3} t_{2} H_{3,2}+t_{3} t_{1} H_{3,1}+t_{3} t_{0} H_{3,0} \\
& +t_{2} t_{3} H_{2,3}+t_{2}^{2} H_{2,2}+t_{2} t_{1} H_{2,1}+t_{2} t_{0} H_{2,0} \\
& +t_{1} t_{3} H_{1,3}+t_{1} t_{2} H_{1,2}+t_{1}^{2} H_{1,1}+t_{1} t_{0} H_{1,0} \\
& +t_{0} t_{3} H_{0,3}+t_{0} t_{2} H_{0,2}+t_{0} t_{1} H_{0,1}+t_{0}^{2} H_{0,0} .
\end{aligned}
$$

Where the $H_{i, j}$ are given by

$$
\begin{aligned}
& H_{3,3}=-a_{0}\left(a_{1}+4 a_{0} \alpha\right), \\
& H_{2,2}=-3 a_{0} a_{3}-a_{1} a_{2}-\alpha\left(3 a_{1}^{2}-2 a_{0} a_{2}\right), \\
& H_{1,1}=-3 a_{1} a_{4}-a_{2} a_{3}-2 \alpha\left(a_{2}^{2}-a_{1} a_{3}-2 a_{0} a_{4}\right), \\
& H_{0,0}=-a_{3} a_{4}-\alpha\left(a_{3}^{2}-2 a_{2} a_{4}\right), \\
& H_{3,2}=H_{2,3}=-2 a_{0} a_{2}-3 a_{0} a_{1} \alpha, \\
& H_{3,1}=H_{1,3}=-3 a_{0} a_{3}-2 a_{0} a_{2} \alpha, \\
& H_{3,0}=H_{0,3}=-4 a_{0} a_{4}-a_{0} a_{3} \alpha, \\
& H_{2,1}=H_{1,2}=-4 a_{0} a_{4}-2 a_{1} a_{3}-\alpha\left(2 a_{1} a_{2}-3 a_{0} a_{3}\right), \\
& H_{2,0}=H_{0,2}=-3 a_{1} a_{4}-\alpha\left(a_{1} a_{3}-4 a_{0} a_{4}\right), \\
& H_{1,0}=H_{0,1}=-2 a_{2} a_{4}-\alpha\left(a_{2} a_{3}-3 a_{1} a_{4}\right) .
\end{aligned}
$$

To help in the determination of the number of negative terms in $H_{\alpha}$, we shall use the determinant of the $H_{i, j}$, which determinant we shall now develop.

\$. The Determinant of Hermite's Form. Let us now set

$$
H=\sum_{0}^{i} \sum_{n=1}^{j} H_{i_{j} j} t_{i} t_{j}
$$

Since $H_{i, j}$ is the coefficient of $t_{i} t_{j}$ we see by (21) and (22) it is given by

$$
H_{i, j}=S(x-\alpha) f_{n-i-1} f_{n-j-1} .
$$

Using this relation we see that the determinant $\Delta^{\prime}$ of the $n^{2}$ quantities $H_{i, j}$, 
that is the determinant of the quadratic form $H$, is the determinant obtained by multiplying the two determinants below by columns,

$$
\begin{aligned}
& \Delta_{n}^{\prime}(\alpha)=\left|\begin{array}{l}
\left(x_{1}-\alpha\right) f_{0}\left(x_{1}\right),\left(x_{1}-\alpha\right) f_{1}\left(x_{1}\right), \cdots,\left(x_{1}-\alpha\right) f_{n-1}\left(x_{1}\right) \\
\left(x_{2}-\alpha\right) f_{0}\left(x_{2}\right),\left(x_{2}-\alpha\right) f_{1}\left(x_{2}\right), \cdots,\left(x_{2}-\alpha\right) f_{n-1}\left(x_{2}\right) \\
\cdot \cdot \cdot \cdot \cdot \cdot \cdot \cdot \cdot \cdot \cdot \cdot \cdot \cdot \cdot \cdot \cdot \cdot \cdot \cdot \cdot \cdot \cdot \cdot \cdot \cdot \cdot \cdot \cdot \cdot f_{n-1}\left(x_{n}\right)
\end{array}\right|
\end{aligned}
$$

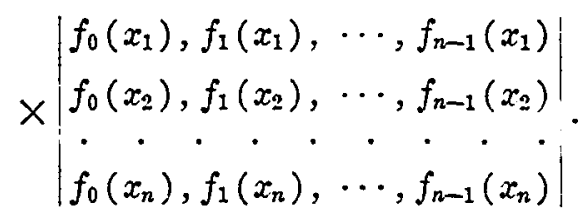

Since $a_{0}\left(x_{1}-\alpha\right)\left(x_{2}-\alpha\right)\left(x_{3}-\alpha\right) \cdots\left(x_{n}-\alpha\right)=(-1)^{n} f(\alpha), \Delta_{n}^{\prime}(\alpha)$ reduces to

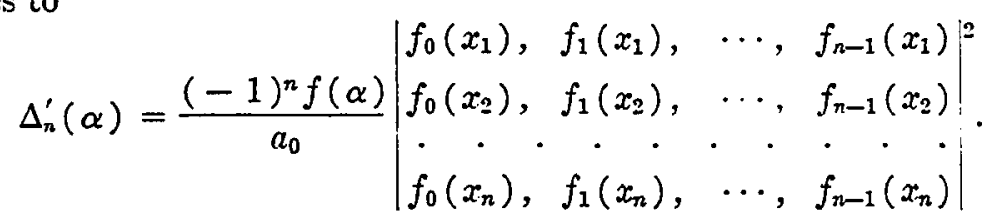

This last determinant may be expressed as the product of two determinants, thus

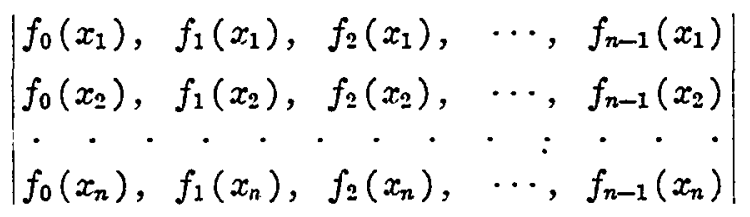

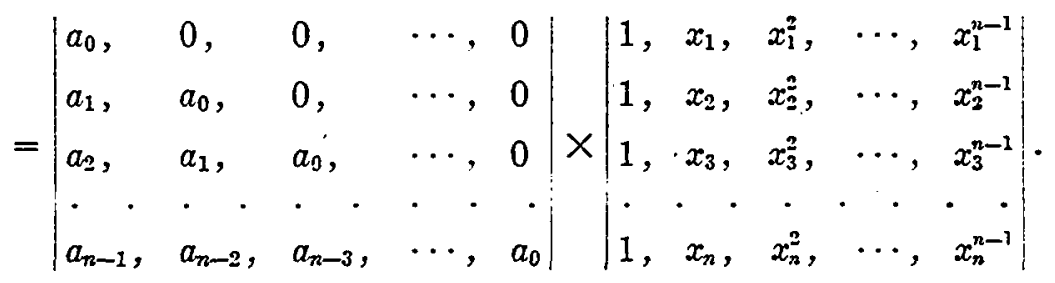

The square of this product is equal to $a_{0}^{2} D, *$ where $D$ is the discriminant of the function $f(x)$. We see then that

$$
\Delta_{n}^{\prime}(\alpha)=(-1)^{n} a_{0} f(\alpha) D
$$

Since, by hypothesis, $D$ is different from zero, $\Delta_{n}^{\prime}(\alpha)$ can only vanish when we substitute one of the roots of $f(x)=0$ for $\alpha$.

Since $\Delta_{n}^{\prime}(\alpha)$ is the determinant of the quadratic form $H$, we can fix the number of negative squares in $H$, if expressed as the sum of squares of linear terms, by the number of variations in the chain of principal minors of $\Delta_{n}^{\prime}(\alpha) \cdot \dagger$ 
By the principal minors of $\Delta_{n}^{\prime}(\alpha)$ we mean determinants formed in succession by starting with $\Delta_{n}^{\prime}(\alpha)$ and leaving off the last row and last column, then leave off the last row and column of this, and so on down to the last. The chain of these principal minors would then appear as

$$
\Delta_{1}^{\prime}(\alpha), \Delta_{2}^{\prime}(\alpha), \Delta_{3}^{\prime}(\alpha), \cdots, \Delta_{n}^{\prime}(\alpha),
$$

when arranged in reverse order.

The number of negative squares in $H$ will then be equal to the number of variations in sign between the functions

$$
1, \Delta_{1}^{\prime}(\alpha), \Delta_{2}^{\prime}(\alpha), \Delta_{3}^{\prime}(\alpha), \cdots, \Delta_{n}^{\prime}(\alpha) .
$$

Since this $H$, as given in (23), is the same as our $H_{a}$ in (5) with the $y$ 's expressed in terms of the $t$ 's, the number of negative squares in $H_{a}$ is obtained from the variations in the signs between the functions in (29). Call this number $N_{a}$, and denote by $N_{\beta}$ the corresponding number of variations in the chain where $\beta$ replaces $\alpha, \beta>\alpha$.

That is $N_{\beta}$ is the number of variations in

$$
1, \Delta_{1}^{\prime}(\beta), \Delta_{2}^{\prime}(\beta), \Delta_{3}^{\prime}(\beta), \cdots, \Delta_{n}^{\prime}(\beta) .
$$

Then $N_{\beta}-N_{\alpha}$ will give us the number of real roots of $f(x)=0$ lying between $\alpha$ and $\beta$. 


\section{CHAPTER III}

\section{The Solution of tee Equation of Secular Variations and Applications}

$\S 1$. The Quadratic Form $\varphi^{\prime}$.-We shall now apply Hermite's method, as outlined above, to the solution of the equation of Secular Variations, which we take in the form:

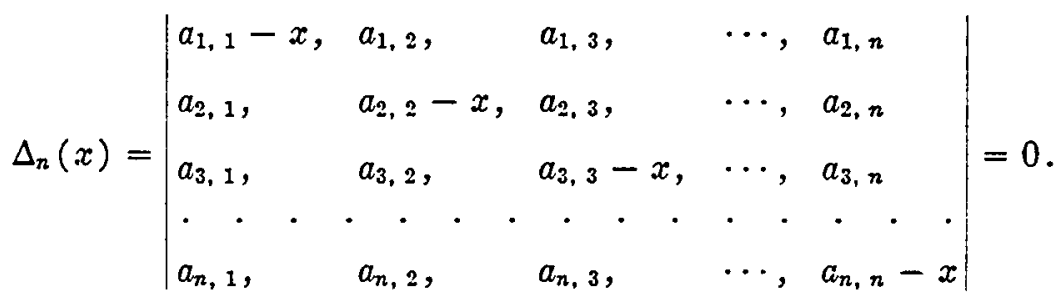

In this we take the $a_{i, ;}$ as real quantities, and further

$$
a_{i, j}=a_{j, i}, \quad \quad \begin{gathered}
i=1,2, \ldots, n . \\
j=1,2, \ldots, n .
\end{gathered}
$$

If this determinant is expanded, we obtain a polynomial of the $n$th degree in $x$, the coefficient of $x^{n}$ being $(-1)^{n}$. Represent this polynomial as

$$
f(x)=a_{0} x^{n}+a_{1} x^{n-1}+a_{2} x^{n-2}+\cdots+a_{n}, \quad a_{0}=(-1)^{n}
$$

and then find the number of roots of

$$
f(x)=0 \text {. }
$$

From (32) form the function $H_{a}$ as shown in (5),

$$
H_{\alpha}=\left(x_{1}-\alpha\right) y_{1}^{2}+\left(x_{2}-\alpha\right) y_{2}^{2}+\cdots+\left(x_{n}-\alpha\right) y_{n}^{2} .
$$

Then the number, $N_{a}$, of negative squares in this function will give the number of real roots of $f(x)=0$, or of $\Delta_{n}(x)=0$, which are less than $\alpha$, plus the number of pairs of imaginary roots of this equation. To enable us to find this quantity $N_{a}$, we shall study with the quadratic form $H_{a}$, another quadratic form whose determinant is $\Delta_{n}(\alpha)$.

Consider the quadratic form

$$
\varphi\left(t_{1}, t_{2}, \cdots, t_{n}\right)=\sum_{\substack{i=1,2, \ldots, n \\ j=1, \frac{2}{2}, \ldots, n}} a_{i_{,}, j} t_{i} t_{j} \quad a_{i, j}=a_{j, i},
$$


whose determinant is

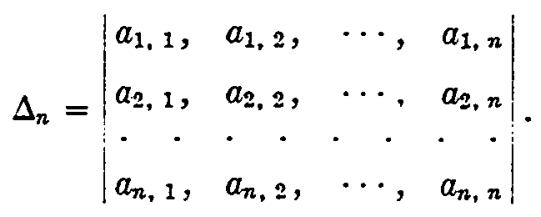

With this form $\varphi$ build the quadratic form,

$$
\varphi^{\prime}=\varphi-\alpha\left(t_{1}^{2}+t_{2}^{2}+\cdots+t_{n}^{2}\right),
$$

the determinant for which is

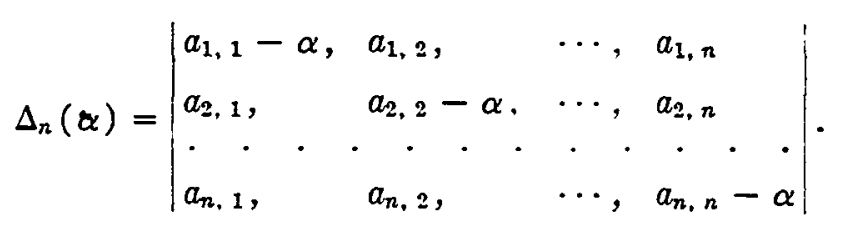

The variations in sign between the terms in the chain

$$
1, \Delta_{1}(\alpha), \Delta_{2}(\alpha), \cdots, \Delta_{n}(\alpha)
$$

will give the number of negative terms of $\varphi^{\prime}$, when expressed as the sum of squares of linear functions.

In what follows we shall assume that no two consecutive terms of this chain (37) vanish for the same value of $x$, and shall later show we are justified in making this assumption. The relation (38) below will help us in determining the number of negative terms in $H_{a}$ from those of the quadratic form $\varphi^{\prime}$,

$$
\Delta_{k}(\alpha) S_{k}-T_{k}^{2}=\Delta_{k-1}(\alpha) \Delta_{k+1}(\alpha) \text {.* }^{*}
$$

In (38) $S_{k}$ and $T_{k}$ are rational integral functions of $x$, and $\Delta_{k}(\alpha), \Delta_{k-1}(\alpha)$ and $\Delta_{k+1}(\alpha)$ have the same meaning as in the chain above.

It is evident from (38) that if $\Delta_{k}(\alpha)=0, \Delta_{k-1}(\alpha)$ and $\Delta_{k+1}(\alpha)$ will have opposite signs.

\$2. Relation between the Negative Squares of $H_{a}$ and $\varphi^{\prime}$. Now let $x$ pass through a real root of $\Delta_{n}(x)=0$, say $x_{r}$. Then take a small quantity, $h$, so small that no root of $\Delta_{n}(x)=0$, excepting $x_{r}$, lies in the interval $x_{r}-h$ to $x_{r}+h$, and let $x_{r}-h=\gamma$ and $x_{r}+h=\delta$. Since $x$ passes through a root of $\Delta_{n}(x)=0$ in going from $\gamma$ to $\delta$ there will be one more negative square in $H_{\delta}$ than in $H_{\gamma} . H_{\delta}$ and $H_{\gamma}$ are used here, as other subscripts will be used, to denote the value of $H_{a}$ in (5) when $\alpha$ is made equal to $\delta$ and $\gamma$ respectively.

Correspondingly if, in the chain of functions (37), we let $x$ pass through the root $x_{r}$, there will be one more variation in sign between the functions of the chain for $x$ slightly larger than $x_{r}$, than there is in the chain for $x$ slightly

* See $\S 88$, page 291, Weber, Vol. I. 
less than $x_{r}$. That is in

$$
1, \Delta_{1}(\delta), \Delta_{2}(\delta), \cdots, \Delta_{n}(\delta)
$$

there is one more variation in sign than there in is the chain

$$
1, \Delta_{1}(\gamma), \Delta_{2}(\gamma), \cdots, \Delta_{n}(\gamma) \text {. }
$$

The fact just stated is true because as $x$ passes through $x_{r} \Delta_{n}(x)$ changes sign, while $\Delta_{n-1}(x)$ does not, and there will be a variation introduced between these two terms in our chain. At the same time there will be no other variation introduced in the chain of functions. If there could be another variation introduced one of the terms, say $\Delta_{p}(x)$, would have to change sign, and hence would have to pass through zero. But as $\Delta_{p}(x)$ passes through zero no variation in sign is introduced since by (38) when $\Delta_{p}(x)=0, \Delta_{p-1}(x)$ and $\Delta_{p+1}(x)$ have opposite signs. $\Delta_{p}(x)$ will then have the same sign as one of them, say $\Delta_{p-1}(x)$ and the opposite sign from the other one $\Delta_{p+1}(x)$, before passing through $x_{r}$, while after passing through $x_{r}, \Delta_{p}(x)$ will have opposite sign to $\Delta_{p-1}(x)$, and same sign as $\Delta_{p+1}(x)$ and will, therefore, keep the number of variations between the three consecutive functions the same. This discussion also shows there will be no variation lost in this interval. There will, therefore, be no variation in sign introduced or lost in the chain of functions as $x$ passes through the root, excepting one variation introduced between $\Delta_{n-1}(x)$ and $\Delta_{n}(x)$. And similarly as $x$ passes through each of the roots of $\Delta_{n}(x)=0$, there will be one variation in sign introduced between the functions of the chain (37).

Since, at the same time, as $x$ increases through each of the roots of

$$
\Delta_{n}(x)=0,
$$

there is one negative square introduced in $H_{a}$, the number of negative squares introduced in $H_{a}$ as $\alpha$ passes from $\alpha$ to $\beta$ will be the same as the number of variations in sign introduced in the chain (37) as $x$ passes from $\alpha$ to $\beta$. Consequently to count the difference in the number of negative squares in $H_{\beta}$ and $H_{a}$ we can find the difference in the number of variations in the two chains

and

$$
1, \Delta_{1}(\beta), \Delta_{2}(\beta), \cdots, \Delta_{n}(\beta)
$$

$$
1, \Delta_{1}(\alpha), \Delta_{2}(\alpha), \cdots, \Delta_{n}(\alpha) \quad \beta>\alpha .
$$

But this is the same as finding the difference in the number of negative squares of $\varphi^{\prime}$, when $\alpha$ is replaced by $\beta$, and in $\varphi^{\prime}$ when $\alpha$ is equal to $\alpha$.

Before we proceed to use the quadratic form $\varphi^{\prime}$ to determine the quantity $N_{\beta}-N_{a}$, which gives us the number of real roots of $\Delta_{n}(x)=0$ lying between $\alpha$ and $\beta$, we shall show we were justified in assuming no two terms of the chain (37) would vanish for the same value of $x$. 
First, it is obvious that if $\Delta_{p-1}(x), \Delta_{p}(x)$ and $\Delta_{p+1}(x)$ all three pass through zero and change sign for the same value of $x$, there will be no change in the variations or permanences in the three functions before and after $x$ passes through this value.

Suppose, then, for some value of $x$ in the interval in question $\Delta_{p}(x)$ and $\Delta_{p+1}(x)$ both vanish, but $\Delta_{p-1}(x)$ does not. In this case we can so choose the $a_{p+1, i}$, which occur in $\Delta_{p+1}(x)$, but not in $\Delta_{p}(x)$, that $\Delta_{p+1}(x)$ will not vanish when $\Delta_{p}(x)$ vanishes. This is true because $\Delta_{p+1}(x)$ cannot vanish identically for all values of $a_{k+1, i}$ since it contains the term $-a_{p+1, p}^{2} \Delta_{p-1}(x)$, * which can only vanish if $a_{p+1, p}=0$.

We can then build a new function $\bar{\Delta}_{n}(x)$, in which the $a_{i, j}^{\prime}$ are chosen to differ by less than an arbitrarily small amount, $\epsilon$, from the corresponding $a_{i, j}$ terms of $\Delta_{n}(x)$, but so that no two consecutive terms in the chain of principal minors of $\bar{\Delta}_{n}(x)$ will vanish for the same values of $x$. $\epsilon$ can be made so small that the roots of $\Delta_{n}(x)=0$ and $\bar{\Delta}_{n}(x)=0$ will differ by less than any assigned arbitrarily small quantity, and consequently a variation will be introduced between $\Delta_{n-1}(x)$ and $\Delta_{n}(x)$, and between $\bar{\Delta}_{n-1}(x)$ and $\bar{\Delta}_{n}(x)$ within the neighborhood of the same quantity. Then the two quantities $\alpha$ and $\beta$ can be so chosen that in the two chains

and

$$
1, \Delta_{1}(x), \Delta_{2}(x), \cdots, \Delta_{n}(x)
$$

$$
1, \bar{\Delta}_{1}(x), \bar{\Delta}_{2}(x), \cdots, \bar{\Delta}_{n}(x)
$$

none of the terms will vanish for $x=\alpha$ or for $x=\beta$. The corresponding members of the two chains will then have the same signs. Since no two consecutive terms of the chain (ii) vanish for the same value of $x$, then the discussion above, regarding the variations in sign introduced as $x$ passes through a root of $\Delta_{n}(x)=0$, holds for this chain. But the difference in the number of variations in sign of the functions of the chains for $x=\beta$ and for $x=\alpha$ will be the same for both chains, and may be determined from either.

The assumption, therefore, that no two consecutive terms of the chain (37) would vanish for the same value of $x$ did not lead to false conclusions, and the quantity $N_{\beta}-N_{\alpha}$ can be found by determining the number of negative squares in $\varphi^{\prime}$ for $x=\alpha$ and $x=\beta$.

This is done by determining the number of variations in signs between the principal minors of its determinant when arranged in the form of the chain (37). Since this at the same time gives the difference in the number of negative squares between $H_{\beta}$ and $H_{a}$, and consequently the number of roots of

$$
\Delta_{n}(x)=0
$$

lying in the interval from $\alpha$ to $\beta$, we shall use it immediately for that purpose.

* See $\$ 62$, "Theory of Determinants," by Thomas Muir. 
§3. Solution of the Equation. Since

$$
\Delta_{p}(\alpha)=(-1)^{p} \alpha^{p}+p_{1} \alpha^{p-1}+p_{2} \alpha^{p-2}+\cdots+p_{n}
$$

$\left(p_{1}, p_{2}, \cdots, p_{n}\right.$ being constants) we note that for $\alpha$ sufficiently large in absolute value, the sign of $\Delta_{p}(\alpha)$ will be the sign of the leading term $(-1)^{p} \alpha^{p}$. In the chain

$$
1, \Delta_{1}(\alpha), \Delta_{2}(\alpha), \cdots, \Delta_{n}(\alpha)
$$

for $\alpha=-\infty$ each member of the chain is positive $\left[(-1)^{p}(-\infty)^{p}\right.$ being positive whether $p$ is even or odd], there are no variations in sign between these members and

$$
N_{-\infty}=0 \text {. }
$$

For $\alpha=+\infty$, on the other hand, there is a variation between each successive term, as the sign of each term, beginning with the second, is determined by $(-1)^{p}$, which makes $\Delta_{1}(+\infty)$ negative and each member of the chain after that alternately positive and negative. There are then $n$ variations in the chain of functions for $\alpha=+\infty$ or

Hence we have

$$
N_{+\infty}=n \text {. }
$$

$$
\text { - } \quad N_{\infty}-N_{-\infty}=n
$$

and we see that all of the roots of the equation of secular variations are real.

$\S 4$. Condition for All of the Roots to be Positive. In order to fix the conditions to be satisfied in order that all the roots of $\Delta_{n}(x)=0$ may be positive, make $\alpha$ take the value $\delta$, where $\delta$ is a positive quantity so small that there are no positive roots of $\Delta_{n}(x)=0$ that are less than $\delta$.

Since the number of variations in sign in the chain of functions $\Delta_{i}(\alpha)$ for $\alpha=+\infty$ is $n$, in order to have all the roots of $\Delta_{n}(x)=0$ positive it is necesșary that, when $\alpha=\delta$, there should be no variations in sign in the functions

$$
1, \Delta_{1}(\delta), \Delta_{2}(\delta), \Delta_{3}(\delta), \cdots, \Delta_{n}(\delta),
$$

that is, they must all be positive. By taking $\delta$ sufficiently small the sign of these functions will be the same as the corresponding principal minors of

or of

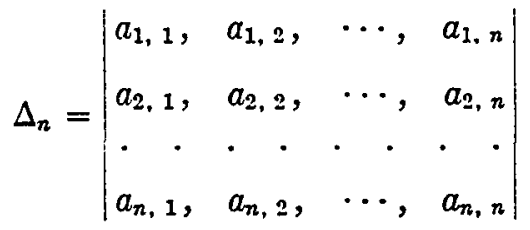

$$
1, \Delta_{1}, \Delta_{2}, \Delta_{3}, \cdots, \Delta_{n}
$$

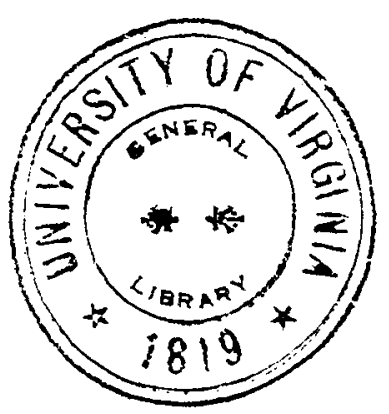


where

$$
\Delta_{1}=a_{1,1}, \quad \Delta_{2}=\left|\begin{array}{ll}
a_{1,1}, & a_{1,2} \\
a_{2,1}, & a_{2,2}
\end{array}\right|, \quad \text { etc. }
$$

Hence if the roots of the given equation $\Delta_{n}(x)=0$ are all to be positive then $\Delta_{1}, \Delta_{2}, \cdots, \Delta_{n}$ must all be positive.

\$5. Principal Planes of Surfaces of Second Degree. As a particular application we shall consider an equation used in finding the principal planes of surfaces of the second degree. The equations of these principal planes depend on the solutions of the equation*

$$
\left|\begin{array}{lll}
a-\lambda, & h, & g \\
h, & b-\lambda, & f \\
g, & f, & c-\lambda
\end{array}\right|=0 .
$$

We see this is the equation $\Delta_{n}(x)=0$ with $n=3$, and it follows, therefore, that its roots are all real.

\$6. Relation between the Roots of $\Delta_{n}(x)=0$ and $\Delta_{n+1}(x)=0$. Let us locate the roots of $\Delta_{n+1}(x)=0$ in relation to those of $\Delta_{n}(x)=0$. Note that, since all the roots of $\Delta_{n}(x)=0$ are real, and there are no variations in sign in the chain (37) for $\alpha=-\infty$, the number of variations in sign of the chain (37) will give the number of roots of $\Delta_{n}(x)=0$ that are less than $\alpha$.

Take for a general investigation $n=r$.

Let the roots of $\Delta_{r}(x)=0$, arranged in order of magnitude, beginning with the smallest, be

$$
\alpha_{1}, \alpha_{2}, \alpha_{3}, \cdots, \alpha_{r-1}, \alpha_{r},
$$

and examine the chains of principal minors of

$$
\Delta_{r}(x) \text { and } \Delta_{r+1}(x) .
$$

In the chain of principal minors

since

$$
1, \Delta_{1}\left(\alpha_{r}\right), \Delta_{2}\left(\alpha_{r}\right), \cdots, \Delta_{r-1}\left(\alpha_{r}\right), \Delta_{r}\left(\alpha_{r}\right), \Delta_{r+1}\left(\alpha_{r}\right)
$$

then

$$
\Delta_{r}\left(\alpha_{r}\right)=0 \text {, }
$$

$$
\Delta_{r-1}\left(\alpha_{r}\right) \quad \text { and } \quad \Delta_{r+1}\left(\alpha_{r}\right)
$$

have opposite signs, by (38). Increase $\alpha_{r}$ by a small amount $h$, where, as previously used, $h$ is a small positive quantity, so small that none of the functions $\Delta_{i}(\alpha)$ will change sign in the interval $\alpha_{r}-h$ to $\alpha_{r}+h$, excepting $\Delta_{r}(\alpha)$.

* See Echols' Calculus, page 361. 
In the two chains of principal minors

(i)

and

$$
1, \Delta_{1}\left(\alpha_{r}\right), \Delta_{2}\left(\alpha_{r}\right), \cdots, \Delta_{r-1}\left(\alpha_{r}\right), \Delta_{r}\left(\alpha_{r}\right)
$$

(ii) $\quad 1, \Delta_{1}\left(\alpha_{r}+h\right), \Delta_{2}\left(\alpha_{r}+h\right), \cdots, \Delta_{r-1}\left(\alpha_{r}+h\right), \Delta_{r}\left(\alpha_{r}+h\right)$

all of the corresponding functions will have the same sign excepting the last one, which in the first chain is zero, but in the second chain may be either positive or negative. The second chain (ii) will have $r$ variations, since all of the roots of $\Delta_{r}(x)=0$ are less than $\alpha_{r}+h$, having taken $\alpha_{r}$ as the greatest root. Having $r$ variations there will be a variation between each pair of successive functions, and consequently a variation between $\Delta_{r-1}\left(\alpha_{r}+h\right)$ and $\Delta_{r}\left(\alpha_{r}+h\right)$. Since $\Delta_{r-1}\left(\alpha_{r}\right)$ and $\Delta_{r+1}\left(\alpha_{r}\right)$ have opposite signs so will $\Delta_{r-1}\left(\alpha_{r}+h\right)$ and $\Delta_{r+1}\left(\alpha_{r}+h\right)$ have opposite signs and from above

$$
\Delta_{r}\left(\alpha_{r}+h\right) \text { and } \Delta_{r+1}\left(\alpha_{r}+h\right)
$$

will have the same sign. Then the chain

(iii) 1, $\Delta_{1}\left(\alpha_{r}+h\right), \Delta_{2}\left(\alpha_{r}+h\right), \cdots, \Delta_{r-1}\left(\alpha_{r}+h\right), \Delta_{r}\left(\alpha_{r}+h\right)$,

$$
\Delta_{r+1}\left(\alpha_{r}+h\right)
$$

will have only $r$ variations, the same as in (ii). Hence there are $r$ roots of $\Delta_{i+1}(x)=0$ less than $\alpha_{r}+h$, and since $\Delta_{r+1}(x)=0$ has $r+1$ roots there is one root of $\Delta_{r+1}(x)=0$ which is greater than $\alpha_{\tau}+h$. That is, there is one, and only one, root of $\Delta_{r+1}(x)=0$ greater than the greatest root of $\Delta_{r}(x)=0$.

Next consider the root $\alpha_{r-1}$, which is the second largest root of $\Delta_{r}(x)=0$. In the chain

$$
1, \Delta_{1}\left(\alpha_{r-1}\right), \Delta_{2}\left(\alpha_{r-1}\right), \cdots, \Delta_{r-1}\left(\alpha_{r-1}\right), \Delta_{r}\left(\alpha_{r-1}\right), \Delta_{r+1}\left(\alpha_{r-1}\right),
$$

since $\Delta_{r}\left(\alpha_{r-1}\right)=0$, then by (38) $\Delta_{r-1}\left(\alpha_{r-1}\right)$ and $\Delta_{r+1}\left(\alpha_{r-1}\right)$ have opposite signs. From this it follows that

$$
\Delta_{r-1}\left(\alpha_{r-1} \pm h\right) \text { and } \Delta_{r+1}\left(\alpha_{r-1} \pm h\right)
$$

have opposite signs, as they have the same sign as $\Delta_{r-1}\left(\alpha_{r-1}\right)$ and $\Delta_{r+1}\left(\alpha_{r-1}\right)$ respectively.

The chain

(iv) $1, \Delta_{1}\left(\alpha_{r-1}+h\right), \Delta_{2}\left(\alpha_{r-1}+h\right), \cdots, \Delta_{r-1}\left(\alpha_{r-1}+h\right), \Delta_{r}\left(\alpha_{r-1}+h\right)$ will have $r-1$ variations, since there are $r-1$ roots of $\Delta_{r}(x)=0$ less than $\alpha_{r-1}+h$. On the other hand there will be only $r-2$ variations in

(v) $1, \Delta_{1}\left(\alpha_{r-1}-h\right), \Delta_{2}\left(\alpha_{r-1}-h\right), \cdots, \Delta_{r-1}\left(\alpha_{r-1}-h\right), \Delta_{r}\left(\alpha_{r-1}-h\right)$.

As there is a variation gained in (iv) over (v) and is gained by the last function 
of the chain, $\Delta_{r}(\alpha)$, changing sign on passing through the root $\alpha_{r-1}$, then in (v) the last two functions have the same sign, but in (iv) they must have opposite signs. That is

$$
\Delta_{r-1}\left(\alpha_{r-1}+h\right) \text { and } \Delta_{r}\left(\alpha_{r-1}+h\right)
$$

will have opposite signs.

In the chain

$$
\begin{gathered}
1, \Delta_{1}\left(\alpha_{r-1}+h\right), \Delta_{2}\left(\alpha_{r-1}+h\right), \cdots, \Delta_{r-1}\left(\alpha_{r-1}+h\right), \\
\Delta_{r}\left(\alpha_{r-1}+h\right), \Delta_{r+1}\left(\alpha_{r-1}+h\right),
\end{gathered}
$$

the functions up to and including $\Delta_{r}\left(\alpha_{r-1}+h\right)$ are the same as in (iv) above, and hence there are $r-1$ variations in this part of the chain (vi) and, in fact, we shall show there are only $r-1$ variations in the chain (vi). There is no variation between $\Delta_{r}\left(\alpha_{r-1}+h\right)$ and $\Delta_{r+1}\left(\alpha_{r-1}+h\right)$, because by $(A)$ $\Delta_{r-1}\left(\alpha_{r-1}+h\right)$ and $\Delta_{r+1}\left(\alpha_{r-1}+h\right)$ have opposite signs, and by $(B)$ $\Delta_{r-1}\left(\alpha_{r-1}+h\right)$ and $\Delta_{r}\left(\alpha_{r-1}+h\right)$ have opposite signs, hence $\Delta_{r}\left(\alpha_{r-1}+h\right)$ and $\Delta_{r+1}\left(\alpha_{r-1}+h\right)$ have same sings. Therefore there are $r-1$ variations in sign in the chain (vi), and therefore $r-1$ roots of $\Delta_{r+1}(x)=0$ less than $\alpha_{\tau-1}+h$, or less than $\alpha_{r-1}$. The remaining two roots of $\Delta_{r+1}(x)=0$ are then greater thail $\alpha_{r-1}$ and since we have shown that one, but only one, of these is greater than $\alpha_{\tau}$, then the other one falls between $\alpha_{\tau-1}$ and $\alpha_{r}$.

Continuing this process it is easy to show that each root in succession of $\Delta_{r}(x)=0$ falls between successive roots of $\Delta_{r+1}(x)=0$, going down in order of magnitude.

Finally for $\alpha_{1}$, in the chain

$$
1, \Delta_{1}\left(\alpha_{1}\right), \Delta_{2}\left(\alpha_{1}\right), \cdots, \Delta_{r-1}\left(\alpha_{1}\right), \Delta_{r}\left(\alpha_{1}\right), \Delta_{r+1}\left(\alpha_{1}\right)
$$

$\Delta_{r-1}\left(\alpha_{1}\right)$ and $\Delta_{r+1}\left(\alpha_{1}\right)$ have opposite signs, since $\Delta_{r}\left(\alpha_{1}\right)=0$.

This is likewise true of $\Delta_{r-1}\left(\alpha_{1} \pm h\right)$ and $\Delta_{r+1}\left(\alpha_{1} \pm h\right)$ as they have the same sign as $\Delta_{r-1}\left(\alpha_{1}\right)$ and $\Delta_{r+1}\left(\alpha_{1}\right)$ respectively.

In the chain

(vii) $1, \Delta_{1}\left(\alpha_{1}-h\right), \Delta_{2}\left(\alpha_{1}-h\right), \cdots, \Delta_{r-1}\left(\alpha_{1}-h\right), \Delta_{r}\left(\alpha_{1}-h\right)$

there are no variations, $\alpha_{1}-h$ being smaller than any root of $\Delta_{r}(x)=0$. However, in the chain

(viii) $1, \Delta_{1}\left(\alpha_{1}+h\right), \Delta_{2}\left(\alpha_{1}+h\right), \cdots, \Delta_{r-1}\left(\alpha_{1}+h\right), \Delta_{r}\left(\alpha_{1}+h\right)$

there is one variation, one root $\alpha_{1}$, of $\Delta_{r}(x)=0$ being less than $\alpha_{1}+h$. Being no variation in (vii), while there is one in (viii), which occurs between the last two functions, then $\Delta_{r-1}\left(\alpha_{1}-h\right)$ and $\Delta_{r}\left(\alpha_{1}-h\right)$ bave the same, sign, while $\Delta_{r-1}\left(\alpha_{1}+h\right)$ and $\Delta_{r}\left(\alpha_{1}+h\right)$ have opposite signs. 
Next in the chain

(ix)

$$
\begin{gathered}
1, \Delta_{1}\left(\alpha_{1}-h\right), \Delta_{2}\left(\alpha_{1}-h\right), \cdots, \Delta_{r-1}\left(\alpha_{1}-h\right), \Delta_{r}\left(\alpha_{1}-h\right), \\
\Delta_{r+1}\left(\alpha_{1}-h\right)
\end{gathered}
$$

the functions up to and including $\Delta_{r}\left(\alpha_{1}-h\right)$ are identical with those in the chain (vii), and there will be no variations in that part of the series (ix). However, since $\Delta_{r-1}\left(\alpha_{1}-h\right)$ and $\Delta_{r+1}\left(\alpha_{1}-h\right)$ have opposite signs, and $\Delta_{r-1}\left(\alpha_{1}-h\right)$ and $\Delta_{r}\left(\alpha_{1}-h\right)$ have same signs, then $\Delta_{r}\left(\alpha_{1}-h\right)$ and $\Delta_{r+1}\left(\alpha_{1}-h\right)$ have opposite signs, there is one variation between the last two functions in $(i x)$ and hence one variation for the chain.

It follows, then, that one, and only one, root of $\Delta_{\tau+1}(x)=0$, is smaller than $\alpha_{1}$ the smallest root of $\Delta_{r}(x)=0$. In the set of equations,

$$
\begin{aligned}
\Delta_{1}(x) & =0, \\
\Delta_{2}(x) & =0, \\
\Delta_{3}(x) & =0, \\
\cdot & \cdot \\
\Delta_{r-1}(x) & =0, \\
\Delta_{r}(x) & =0, \\
\Delta_{r+1}(x) & =0, \\
\cdot & \cdot \\
\Delta_{n-1}(x) & =0, \\
\Delta_{n}(x) & =0,
\end{aligned}
$$

$\Delta_{n}(x)=0$ is our equation of secular variations shown in (30), $\Delta_{n-1}(x)=0$ is found from $\Delta_{n}(x)=0$ by leaving off the last row and column, and so on down for all of the equations to

$$
\Delta_{2}(x)=\left|\begin{array}{cc}
a_{1,1}-x, & a_{1,2} \\
a_{2,1}, & a_{2,2}-x
\end{array}\right|=0
$$

and $\Delta_{1}(x)=a_{1,1}-x=0$, that is, the several equations are the principal minors of $\Delta_{n}(x)$ equated to zero. By our work above, the roots of all of these equations are real, the roots of $\Delta_{n-1}(x)=0$ lie one by one between successive pairs of roots of $\Delta_{n}(x)=0$, the roots of $\Delta_{n-2}(x)=0$ lie similarly between each adjacent pair of roots of $\Delta_{n-1}(x)=0$, and so on down until finally the root of $\Delta_{1}(x)=0$ lies between the two roots of $\Delta_{2}(x)=0$. In other words, the set of equations $(C)$ form what is called limiting equations. ${ }^{*}$

* See Burnside and Panton, 1904, Vol. I, page 187. 
The roots of the equations $(C)$ when indicated by points of a straight line would be represented by the following scheme,

$$
\begin{aligned}
& \Delta_{n}(x)=0 \text {. . . . . . . . . . } n \text { roots } \\
& \Delta_{n-1}(x)=0 \quad \text {. . . . . . . . . } n-1 \text { roots } \\
& \Delta_{n-2}(x)=0 \quad \text {. . . . . . . . n } n-2 \text { roots } \\
& \Delta_{4}(x)=0 \quad \text {. . . . } 4 \text { roots } \\
& \Delta_{3}(x)=0 \quad \text {. . . } 3 \text { roots } \\
& \Delta_{2}(x)=0 \quad \text {. . } 2 \text { roots } \\
& \Delta_{1}(x)=0 \quad \text {. } 1 \text { root }
\end{aligned}
$$

We shall conclude by taking a specific example with numerical coefficients.

$\S 7$. Illustrative Example. Take as illustrative example $n=5$, with the following values for $a_{i, j}$.
$a_{1,1}=2$,
$a_{1,2}=3$,
$a_{1,3}=5$,
$a_{1,4}=6$,
$a_{1,5}=1$,
$a_{2,1}=3, \quad a_{2,2}=3$,
$a_{2,3}=7$,
$a_{2,4}=2$,
$a_{2,5}=3$,
$a_{3,1}=5$,
$a_{3,2}=7$,
$a_{3,3}=1$,
$a_{3,4}=5$,
$a_{3,5}=1$,
$a_{i_{1}}=6$,
$a_{4,2}=2$,
$a_{4,3}=5$,
$a_{4,4}=4$,
$a_{4,5}=2$,
$a_{5,1}=1$,
$a_{5,2}=3$,
$a_{5,3}=1$,
$a_{5,4}=2$,
$a_{5,5}=2$.

This gives

$$
\Delta_{5}(x)=\left|\begin{array}{lllll}
2-x, & 3, & 5, & 6, & 1 \\
3, & 3-x, & 7, & 2, & 3 \\
5, & 7, & 1-x, & 5, & 1 \\
6, & 2, & 5, & 4-x, & 2 \\
1, & 3, & 1, & 2, & 2-x
\end{array}\right|,
$$

from which we see

$$
\begin{aligned}
& \Delta_{5}(x)=-x^{5}+12 x^{4}+108 x^{3}-215 x^{2}-993 x+1217, \\
& \Delta_{4}(x)=x^{4}-10 x^{3}-113 x^{2}+23 x+811, \\
& \Delta_{3}(x)=-x^{3}+6 x^{2}+72 x+34 \\
& \Delta_{2}(x)=x^{2}-5 x-3 \\
& \Delta_{1}(x)=2-x
\end{aligned}
$$


The roots of the equations formed by equating each of these to zero are

Equation

$\Delta_{1}(x)=0$

$\Delta_{2}(x)=0$

$\Delta_{3}(x)=0$

$\Delta_{4}(x) \cdot=0$

$\Delta_{\mathbf{s}}(x)=0$
Roots

2

It is seen here that all the roots are real and that the roots of each equation, one at a time, lie between adjacent pair of roots of the equation which succeeds it. This last fact is more obvious from the diagrammatic representation of the roots shown below.

In the diagram, on the next page, the graphs of the differcnt functions are shown. No attempt has been made to represent the ordinates accurately, on account of the variation in size between the different curves, which would necessitate a different vertical unit for each curve. The relative size of the ordinates are shown and the point of crossing the axis is accurately given. From these points of intersection with the axis of $x$, we see very clearly that the relation between the roots of one equation and those of the equation of next higher degree is that stated above. 
26

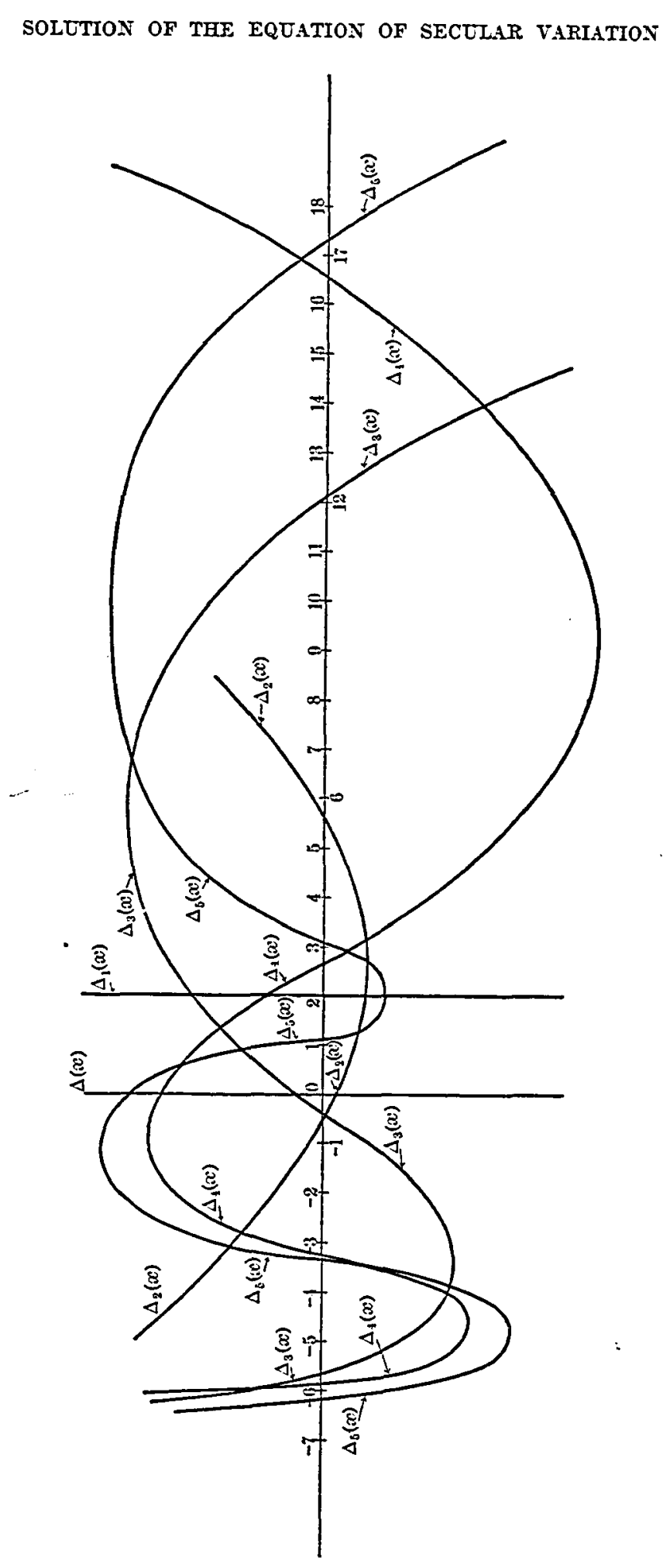

